\title{
Global higher integrability of Jacobians on bounded domains *
}

\author{
by

\section{Jeff HOGAN ${ }^{1}$, Chun LI ${ }^{2}$, Alan McINTOSH ${ }^{3}$, Kewei ZHANG ${ }^{4}$} \\ School of Mathematics, Physics, Computing and Electronics, Macquarie University, \\ Sydney, NSW 2109, Australia
}

Manuscript received 8 September 1998

ABSTRACT. - We give conditions for a vector-valued function $u \in$ $W^{1, n}\left(\Omega, \mathbb{R}^{n}\right)$, satisfying det $D u(x) \geqslant 0$ on a bounded domain $\Omega$, which imply that det $D u(x)$ is globally higher integrable on $\Omega$. We also give conditions for $u \in W^{1, n}\left(\Omega, \mathbb{R}^{n}\right)$ such that det $D u$ belongs to the Hardy space $h_{z}^{1}(\Omega)$ and exhibit some examples which show that our conditions are in some sense optimal. Applications to the weak convergence of Jacobians follow. Div-curl type extensions of these results to forms are also considered.

(C) 2000 L'Association Publications de l'Institut Henri Poincaré. Published by Elsevier B.V. All rights reserved

AMS classification: 42B20, 42B30, 49K99

RÉSUMÉ. - Pour une fonction à valeurs vectorielles $u \in W^{1, n}\left(\Omega, \mathbb{R}^{n}\right)$ telle que det $D u(x) \geqslant 0$ dans un ouvert borné $\Omega$, nous donnons des conditions conduisant à une amélioration de l'intégrabilité globale de $\operatorname{det} D u(x)$ dans un ouvert borné $\Omega$. Nous donnons aussi des conditions sur $u \in W^{1, n}\left(\Omega, \mathbb{R}^{n}\right)$ pour que det $D u$ appartienne à l'espace de Hardy

\footnotetext{
* The authors are supported by the Australian Government through the Australian Research Council.

${ }^{1}$ E-mail: jeffh@mpce.mq.edu.au.

2 E-mail: chun@math.berkeley.edu.

${ }^{3}$ E-mail: alan@mpce.mq.edu.au.

${ }^{4}$ E-mail: kewei@mpce.mq.edu.au.
} 
$h_{z}^{1}(\Omega)$. Quelques exemples démontrent que ces conditions sont dans un certain sens optimales. Ces résultats sont appliqués à la convergence faible des jacobiens. Nous examinons aussi l'extension de ces résultats du type div-curl aux formes différentielles.

(C) 2000 L'Association Publications de l'Institut Henri Poincaré. Published by Elsevier B.V. All rights reserved

\section{INTRODUCTION}

The work of S. Müller [17] has led to many interesting new results regarding important nonlinear quantities such as Jacobians and some quadratic forms in compensated compactness $[12,4]$. However, the results found there are local or interior in nature. For example, Müller's result states that if $u$ is an element of the Sobolev space $W^{1, n}\left(\Omega, \mathbb{R}^{n}\right)\left(\Omega \subset \mathbb{R}^{n}\right)$, and $\operatorname{det} D u(x)=\operatorname{det}\left(\frac{\partial u_{j}}{\partial x_{k}}\right) \geqslant 0$ in $\Omega$ almost everywhere, then for every compact subset $K$ of $\Omega$,

$$
\begin{aligned}
& \int_{K} \operatorname{det} D u(x) \log \left(e+\frac{\operatorname{det} D u(x)}{\int_{\Omega} \operatorname{det} D u(y) d y}\right) d x \\
& \quad \leqslant C(K, n) \int_{\Omega}|D u(x)|^{n} d x .
\end{aligned}
$$

We are interested in finding additional conditions on $u$ under which $\operatorname{det} D u(x) \log (e+\operatorname{det} D u(x))$ is globally integrable on a bounded domain $\Omega$.

Higher integrability results are partly motivated by applications of Jacobians to nonlinear elasticity. A model problem in [7] is that of determining the infimum

$$
\inf _{\left.u\right|_{\partial \Omega}=i d} \int_{\Omega} F(x, \operatorname{det} D u(x)) d x
$$

where $F: \bar{\Omega} \times(0, \infty) \rightarrow[0, \infty)$ is continuous, $\lim _{t \rightarrow 0_{+}} F(x, t)=+\infty$, $\lim _{t \rightarrow+\infty} F(x, t)=+\infty$ and $i d$ is the identity mapping. Let $f: \Omega \rightarrow$ $(0, \infty)$ be a measurable function such that $F(x, f(x))=\min \{F(x, t)$, $t>0\}$ for every fixed $x \in \Omega$. Then the minimizing problem is reduced to solving

$$
\operatorname{det} D u(x)=f(x) \quad \text { in } \Omega, \quad u(x)=x \quad \text { on } \partial \Omega .
$$


This problem is studied in [8] under the condition that $f$ is Hölder continuous, in [24] for $f$ in Sobolev spaces, but has not yet been solved in the case of $f \in L^{p}(\Omega)$. Our global integrability result (Theorem 3.1) gives a necessary condition for the solvability of the above problem for $u \in W^{1, n}\left(\Omega, \mathbb{R}^{N}\right)$. The necessary condition is that $f \log (e+|f|) \in$ $L^{1}(\Omega)$ which we abbreviate by writing $f \in L \log L(\Omega)$.

If $v \in W^{1}\left(\mathbb{R}^{n}, \mathbb{R}\right)$, we denote by $\nabla v$ the vector-valued function $\nabla v=\left(\frac{\partial v}{\partial x_{1}}, \ldots, \frac{\partial v}{\partial x_{n}}\right)$. It was established in [6] that if $u=\left(u_{1}, \ldots, u_{n}\right) \in$ $W^{1, n}\left(\mathbb{R}^{n}, \mathbb{R}^{n}\right)$, then $\operatorname{det} D u$ belongs to the Hardy space $H^{1}\left(\mathbb{R}^{n}\right)$ and $\|\operatorname{det} D u\|_{H^{1}} \leqslant C(n) \prod_{j=1}^{n}\left\|\nabla u_{j}\right\|_{L^{n}}^{n}$. (For relevant details pertaining to the Hardy space $H^{1}\left(\mathbb{R}^{n}\right)$, the reader is referred to Appendix A. Further details can be found in [21].) In [5], Hardy spaces defined on bounded domains $\Omega$ are studied. One such space is

$$
H_{z}^{1}(\Omega):=\left\{f \in L^{1}(\Omega) ; f_{z} \in H^{1}\left(\mathbb{R}^{n}\right)\right\},
$$

where $f_{z}$ is the zero extension of $f$ to $\mathbb{R}^{n}$. Every function $f \in$ $H_{z}^{1}(\Omega)$ satisfies $\int_{\Omega} f(x) d x=0$. The space obtained by removing this cancellation condition is

$$
h_{z}^{1}(\Omega):=\left\{f \in L^{1}(\Omega) ; f-\frac{1}{|\Omega|} \int_{\Omega} f \in H_{z}^{1}(\Omega)\right\} .
$$

Norms on these spaces are defined in the obvious way:

$$
\begin{aligned}
& \|f\|_{H_{z}^{1}(\Omega)}=\left\|f_{z}\right\|_{H^{1}\left(\mathbb{R}^{n}\right)}, \\
& \|f\|_{h_{z}^{1}(\Omega)}=\left\|f-\frac{1}{|\Omega|} \int_{\Omega} f\right\|_{H_{z}^{1}(\Omega)}+\frac{1}{|\Omega|}\left|\int_{\Omega} f\right| .
\end{aligned}
$$

A natural question to ask is: under what conditions on $u \in W^{1, n}\left(\Omega, \mathbb{R}^{n}\right)$ does it follow that $\operatorname{det} D u \in h_{z}^{1}(\Omega)$ ?

In order to solve these problems, in Section 2 we introduce a subspace $K_{\alpha}(\partial \Omega)$ of $W^{1-\frac{1}{n}, n}(\partial \Omega)$ which contains $W^{1-\frac{1}{p}, p}(\partial \Omega)$ for all $p>n$ and which gives better integrability of gradients. We establish our main results under this extra condition. We also discuss the weak continuity of Jacobians on $\Omega$. A crucial element in the proofs is a version of Hölder's inequality adapted to $L \log L(\Omega)$.

In Section 3, we discuss the higher integrability of Jacobians in $L \log L(\Omega)$ by applying the Hardy space result obtained in Section 2. It might be tempting to try to prove this higher integrability by extending $u$ 
to a larger domain $\Omega^{\prime}$ so that the extension is bounded from $W^{1, n}\left(\Omega, \mathbb{R}^{n}\right)$ to $W^{1, n}\left(\Omega^{\prime}, \mathbb{R}^{n}\right)$ and the positivity of det $D u$ is preserved, thus enabling us to use Müller's result to obtain higher integrability on $\Omega$. We show by an example that in general this is not possible.

It is known that questions about Jacobians are special cases of the divcurl problem. In Section 4, we discuss the corresponding Hardy space result, weak continuity and higher integrability for this problem. For the sake of simple notation in describing the extension property, we use the language of differential forms. However, the results obtained on differential forms are interesting in themselves. Many people such as Robbin, Rogers and Temple [19,20] and Iwaniec [11] have considered differential forms in this context.

The authors wish to thank Stephen Montgomery-Smith and Richard O'Neil for several helpful suggestions and references regarding the proof of Proposition 2.2.

\section{HARDY SPACES ON BOUNDED DOMAINS AND WEAK CONTINUITY}

In [6] it is shown that if $u=\left(u_{1}, \ldots, u_{n}\right) \in \dot{W}^{1, n}\left(\mathbb{R}^{n}, \mathbb{R}^{n}\right)$, then the Jacobian det $D u \in H^{1}\left(\mathbb{R}^{n}\right)$, and

$$
\|\operatorname{det} D u\|_{H^{1}\left(\mathbb{R}^{n}\right)} \leqslant C \prod_{j=1}^{n}\left\|\nabla u_{j}\right\|_{L^{n}\left(\mathbb{R}^{n}\right)}^{n} .
$$

Suppose $\Omega$ is a bounded open domain in $\mathbb{R}^{n}$. We are interested in the following question: If $u \in W^{1, n}\left(\Omega, \mathbb{R}^{n}\right)$, is $\operatorname{det} D u \in h_{z}^{1}(\Omega)$ with a similar estimate to that above? The following example from [2] shows that without extra conditions, the answer is negative, even when $\Omega$ is a rectangle in $\mathbb{R}^{2}$.

Example. - Let $n=2$ and $\Omega=(0,2 \pi) \times(-1,1)$. Define a sequence $u^{j}: \bar{\Omega} \rightarrow \mathbb{R}^{2}, j=1,2, \ldots$, by

$$
u^{j}(x, y)=j^{-1 / 2}|y|^{j}(\cos j x, \sin j x) .
$$

Then det $D u^{j}(x, y)=-j y^{2 j-1}$. Thus $\int_{\Omega} \operatorname{det} D u^{j}(x, y)=0$. Notice also that the norms $\left\|D u^{j}\right\|_{L^{2}}$ are bounded. Suppose the estimate holds. Then $\operatorname{det} D u^{j}$ is bounded in $H_{z}^{1}(\Omega)$, and we can extract a subsequence which converges weak-* in $H_{z}^{1}(\Omega)$. On the other hand, det $D u^{j}$ converges 
pointwise to zero. Therefore according to [13], the weak- $*$ limit of the subsequence is also zero. However, for $\phi \in C_{0}^{\infty}\left(\mathbb{R}^{2}\right)$ with $\phi(x, 1)<$ $\phi(x,-1)$,

$$
\begin{gathered}
\liminf _{j \rightarrow \infty} \int_{\mathbb{R}^{2}} \phi(x, y) \operatorname{det} D u^{j}(x, y) d x d y \\
\geqslant \frac{1}{2} \int_{0}^{2 \pi}[\phi(x,-1)-\phi(x, 1)] d x>0,
\end{gathered}
$$

which is a contradiction.

In the sequel, $\Omega$ will denote a bounded open domain in $\mathbb{R}^{n}$ with strongly Lipschitz boundary $\partial \Omega$-an assumption which is enough to ensure

(i) the existence of a bounded extension map from $W^{1, n}(\Omega)$ to $W^{1, n}\left(\mathbb{R}^{n}\right)$, and

(ii) the boundedness of the extension by zero of $W_{0}^{1, n}(\Omega)$ to $W^{1, n}\left(\mathbb{R}^{n}\right)$, where $W_{0}^{1, n}(\Omega)$ is the closure of $C_{0}^{\infty}(\Omega)$ in $W^{1, n}\left(\mathbb{R}^{n}\right)$.

For details, the reader is referred to [1, Section 4]. Although many of the results generalise to non-Lipschitz domains for which these extensions are bounded, we will restrict ourselves to considering domains with strongly Lipschitz boundaries, so that we have concrete realisations of the trace spaces.

In Theorem 2.5 below, we give a sufficient condition under which a modified version of the estimate holds. Before we state the theorem, we introduce some relevant function spaces and state some technical lemmas.

Definition 2.1. - Let $A:[0, \infty) \rightarrow[0, \infty)$ be a monotone increasing function. Under certain technical conditions on A (see [1] and [3]) which are satisfied by all the examples we shall consider in this paper, we consider the Orlicz space $L_{A}(\Omega)$ consisting of (equivalence classes modulo equality a.e. of) measurable functions $f$ on $\Omega$ for which $\int_{\Omega} A(|f(x)|) d x<\infty$. The functional

$$
\|f\|_{A}=\inf \left\{k^{-1} ; k>0 \text { and } \int_{\Omega} A(k|f(x)|) d x \leqslant 1\right\}
$$

is then a norm (the Luxemburg norm) on $L_{A}(\Omega)$ under which it becomes a rearrangement-invariant Banach space. 
When $A(t)=t^{p}(\log (e+t))^{\alpha}(1 \leqslant p<\infty, \alpha \geqslant 0) L_{A}(\Omega)$ is referred to as $L^{p}(\log L)^{\alpha}(\Omega)$ and the associated norm is written $\|f\|_{L^{p}(\log L)^{\alpha}(\Omega)}$. The spaces $L^{p}(\log L)^{0}(\Omega)$ and $L^{1}(\log L)^{1}(\Omega)$ are usually referred to as $L^{p}(\Omega)$ and $L \log L(\Omega)$ respectively.

Since $\Omega$ is bounded, an argument based on rearrangements, maximal functions and Hardy inequalities can be used to prove the equivalence

$$
\|f\|_{L^{p}(\log L)^{\alpha}(\Omega)} \approx\left(\int_{\Omega}|f(x)|^{p}\left(\log \left(e+\frac{|f(x)|}{\|f\|_{p}}\right)\right)^{\alpha} d x\right)^{1 / p} .
$$

Hence the quantity on the left-hand-side of (1.1) (Müller's result) is equivalent to $\|\operatorname{det} D u\|_{L \log L(K)}$. The following generalised Hölder inequality, the proof of which is deferred to Appendix A, will be a crucial element in the proofs of many of our results.

PROPOSITION 2.2. - Let $1<p, q<\infty, \alpha, \beta>0,1 / p+1 / q=1 / r$, $\alpha / p+\beta / q=\gamma / r$ and $f \in L^{p}(\log L)^{\alpha}(\Omega), g \in L^{q}(\log L)^{\beta}(\Omega)$. Then $f g \in L^{r}(\log L)^{\gamma}(\Omega)$ and

$$
\|f g\|_{L^{r}(\log L)^{\gamma}(\Omega)} \leqslant c\|f\|_{L^{p}(\log L)^{\alpha}(\Omega)}\|g\|_{L^{q}(\log L)^{\beta}(\Omega)} .
$$

Remark. - The case $\alpha=\beta=\gamma$ is presented in Lemma 4.2 of [10].

In the proof of our main result, we need the following lemma, the first part of which is a consequence of [23, Chapter I, Section 5.2] (a statement of which appears in Appendix A as Lemma A.3) while the second part is an immediate corollary of the first.

Lemma 2.3. - (i) Suppose $f \in L(\log L)(\Omega)$, and $\int_{\Omega} f=0$. Then $f \in H_{z}^{1}(\Omega)$ and

$$
\|f\|_{H_{z}^{1}(\Omega)} \leqslant c\|f\|_{L(\log L)(\Omega)} .
$$

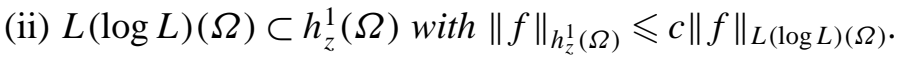

By the Trace Theorem, $\operatorname{Tr} W^{1, n}(\Omega)=W^{1-\frac{1}{n}, n}(\partial \Omega)$. For $\alpha \geqslant 0$, we define a subspace $K_{\alpha}(\partial \Omega)$ of $W^{1-\frac{1}{n}, n}(\partial \Omega)$ as follows.

DEFINITION 2.4. - Let $\alpha>0$. For $\phi \in W^{1-\frac{1}{n}, n}(\partial \Omega)$, we say that $\phi \in K_{\alpha}(\partial \Omega)$ if $\phi$ can be extended into $\Omega$ as $v \in W^{1, n}(\Omega)$ so that $\frac{\partial v}{\partial x_{j}} \in L^{n}(\log L)^{\alpha}(\Omega)$ for $1 \leqslant j \leqslant n$. 
Remark. - Clearly,

$$
W^{1-\frac{1}{p}, p}(\partial \Omega) \subset K_{\alpha}(\partial \Omega) \subset K_{0}(\partial \Omega)=W^{1-\frac{1}{n}, n}(\partial \Omega)
$$

for all $p>n$. Moreover, in [14], $K_{\alpha}(\partial \Omega)$ is realised as the class of those $u \in L^{n}(\log L)^{\alpha}(\partial \Omega)$ for which

$$
\int_{\partial \Omega} \int_{\partial \Omega} \Phi\left(\frac{|u(x)-u(y)|}{|x-y|}\right) \frac{d x d y}{|x-y|^{n-2}}<\infty,
$$

where $\Phi(t)=t^{n}(\log (e+t))^{\alpha}$. It follows that $K_{\alpha}(\partial \Omega) \varsubsetneqq W^{1-\frac{1}{n}, n}(\partial \Omega)$ when $\alpha>0$. Actually, we shall not make use of this realisation. Instead, we define the following semi-norm on $K_{\alpha}(\partial \Omega)$ :

$$
\|\phi\|_{K_{\alpha}}=\inf \left\{\|\nabla v\|_{L^{n}(\log L)^{\alpha}(\Omega)},\left.v\right|_{\partial \Omega}=\phi\right\} .
$$

We come now to the main theorem of this section.

TheOREM 2.5. - Suppose $u \in W^{1, n}\left(\Omega, \mathbb{R}^{n}\right),\left.u_{j}\right|_{\text {д } \Omega} \in K_{\alpha_{j}}(\partial \Omega), j=$ $1,2, \ldots, n$, for $\alpha_{j} \geqslant 0$ and $\sum_{j=1}^{n} \alpha_{j}=n$. Then $\operatorname{det} D u \in h_{z}^{1}(\Omega)$ and

$$
\|\operatorname{det} D u\|_{h_{z}^{1}(\Omega)} \leqslant C \prod_{j=1}^{n}\left(\left\|\nabla u_{j}\right\|_{L^{n}(\Omega)}+\left\|\left.u_{j}\right|_{\partial \Omega}\right\|_{K_{\alpha_{j}}(\partial \Omega)}\right) \text {. }
$$

Remark. - In the case when $\alpha_{1}=n, \alpha_{j}=0$ for $j \geqslant 2$, we have $\operatorname{det} D u \in h_{z}^{1}(\Omega)$ under the single restriction $\left.u_{1}\right|_{\partial \Omega} \in K_{n}(\partial \Omega)$. In the case when $\alpha_{j}=1$ for all $j$, we have $\operatorname{det} D u \in h_{z}^{1}(\Omega)$ and

$$
\|\operatorname{det} D u\|_{h_{z}^{1}(\Omega)} \leqslant C\left(\|D u\|_{L^{n}(\Omega)}+\max _{j}\left\|\left.u_{j}\right|_{\partial \Omega}\right\|_{K_{1}(\partial \Omega)}\right)^{n} .
$$

Remark. - Clearly the boundary condition is satisfied in the important special case $u(x)=x$ on $\partial \Omega$, mentioned in the introduction.

Proof of Theorem 2.5. - Assume without loss of generality that $\int_{\Omega} u_{j}=0$ for each $j$. Since $\Omega$ has a strongly Lipschitz boundary, we can extend $u_{j}$ to $\mathbb{R}^{n}$ so that

$$
\left\|\nabla u_{j}\right\|_{L^{n}\left(\mathbb{R}^{n}\right)} \leqslant\left\|u_{j}\right\|_{W^{1, n}\left(\mathbb{R}^{n}\right)} \leqslant c\left\|u_{j}\right\|_{W^{1, n}(\Omega)} \leqslant c\left\|\nabla u_{j}\right\|_{L^{n}(\Omega)} .
$$

(The last inequality comes from an application of the Poincaré inequality and the assumption that $\int_{\Omega} u_{j}=0$ for each $j$. For details on Poincaré and 
the extension result which provides the second inequality, the reader is referred to [1].) Since $\left.u_{j}\right|_{\partial \Omega} \in K_{\alpha_{j}}(\partial \Omega)$, we can choose $v_{j} \in W^{1, n}\left(\mathbb{R}^{n}\right)$ with

$$
\left\|\nabla v_{j}\right\|_{L^{n}(\log L)^{\alpha_{j}(\Omega)}} \leqslant C\left\|\left.u_{j}\right|_{\partial \Omega}\right\|_{K_{\alpha_{j}}(\partial \Omega)},
$$

and $v_{j}=u_{j}$ on $\mathbb{R}^{n} \backslash \Omega$. (We have used the fact that $\left(v_{j}-u_{j}\right)_{z} \in W^{1, n}\left(\mathbb{R}^{n}\right)$ since $\left.\left(v_{j}-u_{j}\right)\right|_{\partial \Omega}=0$.) Note that

$$
\begin{aligned}
\left\|\nabla v_{j}\right\|_{L^{n}\left(\mathbb{R}^{n}\right)} & \leqslant\left\|\nabla u_{j}\right\|_{L^{n}\left(\mathbb{R}^{n}\right)}+\left\|\nabla v_{j}\right\|_{L^{n}(\Omega)} \\
& \leqslant c\left(\left\|\nabla u_{j}\right\|_{L^{n}(\Omega)}+\left\|\left.u_{j}\right|_{\partial \Omega}\right\|_{K_{\alpha_{j}}(\partial \Omega)}\right) .
\end{aligned}
$$

If we now put

$$
\begin{aligned}
w_{(1)} & =\left(u_{1}-v_{1}, u_{2}, \ldots, u_{n}\right) \\
w_{(2)} & =\left(v_{1}, u_{2}-v_{2}, \ldots, u_{n}\right) \\
& \vdots \\
w_{(n)} & =\left(v_{1}, v_{2}, \ldots, u_{n}-v_{n}\right),
\end{aligned}
$$

then $w_{(k)} \in W^{1, n}\left(\mathbb{R}^{n}, \mathbb{R}^{n}\right)$, and

$$
\operatorname{det} D u=\operatorname{det} D w_{(1)}+\operatorname{det} D w_{(2)}+\cdots+\operatorname{det} D w_{(n)}+\operatorname{det} D v .
$$

On applying (2.1) we see that det $D w_{(k)} \in H^{1}\left(\mathbb{R}^{n}\right)$ and

$$
\begin{aligned}
\left\|\operatorname{det} D w_{(k)}\right\|_{H^{1}\left(\mathbb{R}^{n}\right)} & \leqslant c \prod_{j=1}^{n}\left\|\nabla w_{(k) j}\right\|_{L^{n}\left(\mathbb{R}^{n}\right)} \\
& \leqslant c \prod_{j=1}^{n}\left(\left\|\nabla u_{j}\right\|_{L^{n}(\Omega)}+\left\|\left.u_{j}\right|_{\partial \Omega}\right\|_{K_{\alpha_{j}}(\partial \Omega)}\right) .
\end{aligned}
$$

Since $u_{k}-v_{k}=0$ outside $\Omega$, the support of $D w_{(k)}$ is contained in $\Omega$. Thus det $D w_{(k)} \in H_{z}^{1}(\Omega)$ and

$$
\begin{aligned}
\left\|\operatorname{det} D w_{(k)}\right\|_{h_{z}^{1}(\Omega)} & =\left\|\operatorname{det} D w_{(k)}\right\|_{H_{z}^{1}(\Omega)}=\left\|\operatorname{det} D w_{(k)}\right\|_{H^{1}\left(\mathbb{R}^{n}\right)} \\
& \leqslant c \prod_{j=1}^{n}\left(\left\|\nabla u_{j}\right\|_{L^{n}(\Omega)}+\left\|\left.u_{j}\right|_{\partial \Omega}\right\|_{K_{\alpha_{j}}(\partial \Omega)}\right) .
\end{aligned}
$$

Now consider the final term in (2.2). As a consequence of Proposition 2.2 and Lemma 2.3, we have

$$
\|\operatorname{det} D v\|_{h_{z}^{1}(\Omega)} \leqslant c\|\operatorname{det} D v\|_{L \log L(\Omega)} \leqslant c \prod_{j=1}^{n}\left\|\nabla v_{j}\right\|_{L^{n}(\log L)^{\alpha_{j}}(\Omega)}
$$




$$
\leqslant c \prod_{j}\left\|\left.u_{j}\right|_{\partial \Omega}\right\|_{K_{\alpha_{j}}(\Omega)} .
$$

Combining (2.2), (2.3) and (2.4) completes the proof of Theorem 2.5.

Now we discuss the weak convergence of Jacobians. Suppose $\left\{u^{(k)}\right\} \subset$ $W^{1, n}\left(\Omega, \mathbb{R}^{n}\right)$ is a bounded sequence whose components $\left\{\left.u_{j}^{(k)}\right|_{\partial \Omega}\right\}(j=$ $1,2, \ldots, n)$ are bounded in $K_{\alpha_{j}}(\partial \Omega)$ with $\alpha_{j}$ as in Theorem 2.5. Then we have

$$
\begin{aligned}
& \left\|\operatorname{det} D u^{(k)}-\int_{\Omega} \operatorname{det} D u^{(k)}\right\|_{H_{z}^{1}(\Omega)} \\
& \quad \leqslant C \prod_{j=1}^{n}\left(\left\|\nabla u_{j}^{(k)}\right\|_{L^{n}(\Omega)}+\left\|\left.u_{j}^{(k)}\right|_{\partial \Omega}\right\|_{K_{\alpha_{j}}(\partial \Omega)}\right) \leqslant C^{\prime} .
\end{aligned}
$$

Let

$$
\operatorname{VMO}(\Omega)=\left\{\left.b\right|_{\Omega}: b \in \operatorname{VMO}\left(\mathbb{R}^{n}\right)\right\} .
$$

Then since $\operatorname{VMO}\left(\mathbb{R}^{n}\right)^{*}=H^{1}\left(\mathbb{R}^{n}\right)$, there exists $g \in H_{z}^{1}(\Omega)$ such that for all $b \in \operatorname{VMO}(\Omega)$,

$$
\int_{\Omega} b\left(\operatorname{det} D u^{(k)}-\int_{\Omega} \operatorname{det} D u^{(k)}\right) \rightarrow \int_{\Omega} b g
$$

up to a subsequence. We can suppose that for such a subsequence,

$$
\int_{\Omega} \operatorname{det} D u^{(k)} \rightarrow \delta \in \mathbb{R} .
$$

Therefore, since $b \in \operatorname{VMO}(\Omega) \subset L^{1}(\Omega)$, we have

$$
\int_{\Omega} b\left(\int_{\Omega} \operatorname{det} D u^{(k)}\right) \rightarrow \delta \int_{\Omega} b .
$$

Thus up to a subsequence,

$$
\int_{\Omega} b \operatorname{det} D u^{(k)} \rightarrow \int_{\Omega} b(g+\delta)
$$

for all $b \in \operatorname{VMO}(\Omega)$, where $g+\delta \in h_{z}^{1}(\Omega)$.

The following theorem shows that if furthermore, $u^{(k)} \rightarrow u$ weakly in $W^{1, n}(\Omega)$, then $g+\delta=\operatorname{det} D u$. 
THEOREM 2.6. - Suppose $u^{(k)} \in W^{1, n}\left(\Omega, \mathbb{R}^{n}\right)$ is a bounded sequence for which $\left.u_{j}^{(k)}\right|_{\partial \Omega} \in K_{\alpha_{j}}(\partial \Omega)(j=1,2 \ldots, n)$ is also bounded, $\alpha_{j} \geqslant 0$ and $\sum_{j=1}^{n} \alpha_{j}=n$. Suppose further that $u^{(k)} \rightarrow u$ weakly in $W^{1, n}\left(\Omega, \mathbb{R}^{n}\right)$. Then up to a subsequence,

$$
\int_{\Omega} b \operatorname{det} D u^{(k)} \rightarrow \int_{\Omega} b \operatorname{det} D u
$$

for all $b \in \operatorname{VMO}(\Omega)$.

Proof. - As discussed before, we can suppose that for any $b \in$ $\operatorname{VMO}(\Omega)$,

$$
\int_{\Omega} b\left(\operatorname{det} D u^{(k)}-\int_{\Omega} \operatorname{det} D u^{(k)}\right) \rightarrow \int_{\Omega} b g
$$

for some $g \in H_{z}^{1}(\Omega)$. We first prove that $g=\operatorname{det} D u-\int_{\Omega} \operatorname{det} D u$. As in the proof of Theorem 2.5 , extend $u^{(k)}$ to all of $\mathbb{R}^{n}$, and let $v^{(k)}$ be the chosen function corresponding to $u^{(k)}$. Then up to a subsequence, we can suppose $u^{(k)} \rightarrow \tilde{u}$ weakly in $W^{1, n}\left(\mathbb{R}^{n}, \mathbb{R}^{n}\right)$ and $v^{(k)} \rightarrow v$ weakly in $W^{1, n}\left(\mathbb{R}^{n}, \mathbb{R}^{n}\right)$ for some $\tilde{u}$ and $v$. By uniqueness, $\tilde{u}=u$ on $\Omega$. Let $w_{(j)}^{(k)}$ and $w_{(j)}$ be the functions corresponding to $u^{(k)}$ and $u$ as in the proof of Theorem 2.5. Then

$$
\operatorname{det} D u^{(k)}=\operatorname{det} D w_{(1)}^{(k)}+\operatorname{det} D w_{(2)}^{(k)}+\cdots+\operatorname{det} D w_{(n)}^{(k)}+\operatorname{det} D v^{(k)}
$$

and

$$
\operatorname{det} D u=\operatorname{det} D w_{(1)}+\operatorname{det} D w_{(2)}+\cdots+\operatorname{det} D w_{(n)}+\operatorname{det} D v .
$$

It is easy to see that $w_{(j)}^{(k)} \rightarrow w_{(j)}$ weakly in $W^{1, n}\left(\mathbb{R}^{n}, \mathbb{R}^{n}\right)$. Since $\operatorname{supp} w_{(j)}^{(k)}$, supp $w_{(j)} \subset \bar{\Omega}$, by Corollary IV.1 of [6], for any $b \in \operatorname{VMO}\left(\mathbb{R}^{n}\right)$, then up to a subsequence,

$$
\int_{\Omega} b \operatorname{det} D w_{(j)}^{(k)}=\int_{\mathbb{R}^{n}} b \operatorname{det} D w_{(j)}^{(k)} \rightarrow \int_{\mathbb{R}^{n}} b \operatorname{det} D w_{(j)}=\int_{\Omega} b \operatorname{det} D w_{(j)} .
$$

As for det $D v^{(k)}$, we can assume that the $v^{(k)}$ we choose are supported in a compact set $\Omega_{k} \supset \Omega$, and $D v^{(k)} \in L^{n}(\log L)^{\alpha_{j}}\left(\mathbb{R}^{n}\right)$ are uniformly bounded. Then $\operatorname{det} D v^{(k)}$ are uniformly bounded in $L \log L(\Omega)$. By the criteria of de La Vallée Poussin [9], there is a subsequence of 
$\operatorname{det} D v^{(k)}$ which converges weakly in $L^{1}\left(\Omega_{k}\right)$. Suppose $\operatorname{det} D v^{(k)}$ is such a convergent sequence (otherwise replace it by a subsequence).

Moreover, again by Corollary IV.1 of [6], det $D v^{(k)}$ has a subsequence which converges to det $D v$ weak-* in $H^{1}\left(\mathbb{R}^{n}\right)$. Again replace $\operatorname{det} D v^{(k)}$ by such a convergent subsequence. Then we have that det $D v^{(k)}$ converges weakly in $L^{1}\left(\Omega_{k}\right)$ to some function $h$ and $\operatorname{det} D v^{(k)}$ converges weak-* in $H^{1}\left(\mathbb{R}^{n}\right)$ to det $D v$. Since both convergences imply the convergence in the distributional sense on $\mathbb{R}^{n}$, by uniqueness of the limit we have $h=\operatorname{det} D v$ (taking $h$ a function on $\mathbb{R}^{n}$ with compact support in $\Omega_{k}$ ).

Thus, we have shown that $\operatorname{det} D v^{(k)}$ converges to $\operatorname{det} D v$ weakly in $L^{1}\left(\Omega_{k}\right)$ and thus also weakly in $L^{1}(\Omega)$. Combining the above results we get, for $b \in C(\bar{\Omega}) \subset L^{\infty}(\Omega) \cap \operatorname{VMO}(\Omega)$,

$$
\begin{aligned}
\int_{\Omega} b \operatorname{det} D u^{(k)} & =\int_{\Omega} b \sum_{j=1}^{n} \operatorname{det} D w_{j}^{(k)}+\int_{\Omega} b \operatorname{det} D v^{(k)} \\
& \rightarrow \int_{\Omega} b \sum_{j=1}^{n} \operatorname{det} D w_{j}+\int_{\Omega} b \operatorname{det} D v=\int_{\Omega} b \operatorname{det} D u .
\end{aligned}
$$

Therefore, for $b \in C(\bar{\Omega})$,

$$
\int_{\Omega} b\left(\operatorname{det} D u^{(k)}-\int_{\Omega} \operatorname{det} D u^{(k)}\right) \rightarrow \int_{\Omega} b\left(\operatorname{det} D u-\int_{\Omega} \operatorname{det} D u\right),
$$

which implies $g=\operatorname{det} D u-\int_{\Omega} \operatorname{det} D u$.

Thus, for any $b \in \operatorname{VMO}(\Omega)$,

$$
\int_{\Omega} b \operatorname{det} D u^{(k)} \rightarrow \int_{\Omega} b \operatorname{det} D u,
$$

and the proof is completed.

As a corollary of this theorem, we have the following result.

COROLlary 2.7. - Suppose $u^{(k)} \in W^{1, n}(\Omega)$ is a bounded sequence, $u^{(k)} \rightarrow u$ weakly in $W^{1, n}(\Omega)$ and $\left.\left(u^{(k)}-u\right)\right|_{\partial \Omega} \in K_{n}(\partial \Omega)$ is also bounded. Then up to a subsequence,

$$
\int_{\Omega} b \operatorname{det} D u^{(k)} \rightarrow \int_{\Omega} b \operatorname{det} D u
$$

for all $b \in \operatorname{VMO}(\Omega)$. 
Remark. - In [11], Iwaniec proves the same result under the stronger assumptions that $\left.\left(u^{(k)}-u\right)\right|_{\partial \Omega}=0$ and that $\Omega$ has a smooth boundary.

Proof of Corollary 2.7. - For each $k$, write

$$
\operatorname{det} D u^{(k)}=\sum_{j=1}^{n} \operatorname{det} D w_{(j)}^{(k)}+\operatorname{det} D u
$$

where

$$
w_{(j)}^{(k)}=\left(u_{1}, \ldots, u_{j-1}, u_{j}^{(k)}-u_{j}, u_{j+1}^{(k)}, \ldots, u_{n}^{(k)}\right) .
$$

Apply Theorem 2.6 with $\alpha_{j}=n$ and $\alpha_{i}=0$ for $i \neq j$ to see that, up to a subsequence,

$$
\int_{\Omega} b \operatorname{det} D w_{(j)}^{(k)} \rightarrow 0
$$

for all $b \in \operatorname{VMO}(\Omega)$. Therefore,

$$
\int_{\Omega} b \operatorname{det} D u^{(k)} \rightarrow \int_{\Omega} b \operatorname{det} D u
$$

\section{GLOBAL HIGHER INTEGRABILITY OF JACOBIANS ON $\Omega$}

As seen in [17], for $u \in W^{1, n}\left(\Omega, \mathbb{R}^{n}\right)$ with det $D u \geqslant 0$ on $\Omega$, we have the interior estimate $\operatorname{det} D u \in L \log L(K)$ for compact subsets $K \subset \Omega$. One may think that given some control on the boundary value of $u$, it should be possible to obtain global higher integrability. In this section we show that this is indeed the case, and also show that in some sense the boundary condition we give is optimal.

THEOREM 3.1. - Suppose $u \in W^{1, n}\left(\Omega, \mathbb{R}^{n}\right)$ and $\operatorname{det} D u(x) \geqslant 0$ on $\Omega$. If furthermore $\left.u_{j}\right|_{\partial \Omega} \in K_{\alpha_{j}}(\partial \Omega), j=1,2, \ldots, n$, for $\alpha_{j} \geqslant 0$ and $\sum_{j=1}^{n} \alpha_{j}=n$, then $\operatorname{det} D u \in L \log L(\Omega)$ and

$$
\|\operatorname{det} D u\|_{L \log L(\Omega)} \leqslant c \prod_{j=1}^{n}\left(\left\|\nabla u_{j}\right\|_{L^{n}(\Omega)}+\left\|\left.u_{j}\right|_{\partial \Omega}\right\|_{K_{\alpha_{j}}(\partial \Omega)}\right) \text {. }
$$

This is an immediate consequence of Theorem 2.5 and the following result, which is a partial converse to Lemma 2.3. 
Proposition 3.2. - Suppose $f \in h_{z}^{1}(\Omega)$ and $f \geqslant 0$ on $\Omega$. Then $f \in L \log L(\Omega)$ and

$$
\|f\|_{L \log L(\Omega)} \leqslant c_{\Omega}\|f\|_{h_{z}^{1}(\Omega)} .
$$

The proof of Proposition 3.2 relies on a few well-known properties of maximal functions and Hardy spaces. We defer the proof to Appendix A.

The next result demonstrates that in certain circumstances, the boundary condition of Theorem 3.1 is necessary.

THEOREM 3.3. - Let $\Omega \subset \mathbb{R}^{2}$ be a bounded open domain with Lipschitz boundary and $U=\left(u_{1}, u_{2}\right) \in W^{1,2}(\Omega)$ with $h(z)=u_{1}+i u_{2}$ analytic on $\Omega$. Then $\operatorname{det} D U \in L \log L(\Omega)$ if and only if $\left.u_{j}\right|_{\partial \Omega} \in$ $K_{1}(\partial \Omega)$.

Proof. - Since $u_{1}$ and $u_{2}$ satisfy the Cauchy-Riemannn equations, we have det $D U=\left|h^{\prime}(z)\right|^{2}=\left|\nabla u_{1}\right|^{2}=\left|\nabla u_{2}\right|^{2} \geqslant 0$. If $\operatorname{det} D U \in L \log L(\Omega)$, then $\nabla u_{j} \in L^{2} \log L(\Omega)$, so $\left.u_{j}\right|_{\partial \Omega} \in K_{1}(\partial \Omega)$. The converse is an immediate consequence of Theorem 3.1 with $n=2$ and $\alpha_{1}=\alpha_{2}=1$.

As mentioned in the introduction, Müller's result (1.1) is interior in nature. One might hope that by extending $u$ to a larger domain $\Omega^{\prime}$ in such a way that the extension $\tilde{u}$ satisfies $\operatorname{det} D \tilde{u} \geqslant 0$ on $\Omega^{\prime}$ and $\|\tilde{u}\|_{W^{1,2}\left(\Omega^{\prime}\right)} \leqslant$ $c\|u\|_{W^{1,2}(\Omega)}$, one might obtain a global result on $\Omega$ by applying (1.1) on $\Omega^{\prime}$. The following example shows that in general this is not possible.

Example. - We specialise the situation in Theorem 3.3 to the case where $\Omega=\mathbb{D}=\{z \in \mathbb{C} ;|z|<1\}$ is the unit disc in $\mathbb{C}$ and $\partial \Omega=\mathbb{T}=$ $\{z \in \mathbb{C} ;|z|=1\}$ is its boundary, the unit circle.

Choose $\varphi \in W^{1 / 2,2}(\mathbb{T}) \backslash K_{1}(\mathbb{T})$ real-valued. (This choice is possible by the remark after Definition 2.4.) Then $\varphi$ admits the Fourier series expansion $\varphi\left(e^{i \theta}\right)=\sum_{n=-\infty}^{\infty} c_{n} e^{i n \theta}$ with $c_{-n}=\overline{c_{n}}$ for all $n$ and $\sum_{n=-\infty}^{\infty}(|n|+$ 1) $\left|c_{n}\right|^{2}<\infty$. Define $h(z)=\sum_{n=0}^{\infty} c_{n} z^{n}$. Then $h=u_{1}+i u_{2}$ is an analytic function on $\mathbb{D}, U=\left(u_{1}, u_{2}\right) \in W^{1,2}\left(\Omega, \mathbb{R}^{2}\right)$, and $\left.u_{1}\right|_{\partial \Omega}=\varphi$. Moreover,

$$
\operatorname{det} D U=\left|h^{\prime}(z)\right|^{2}=\left|\nabla u_{1}\right|^{2} \geqslant 0
$$

as in the proof of Theorem 3.3. Suppose now that we could extend $U$ to $\tilde{U}$ on $\Omega \supset \mathbb{D}$, an open set in $\mathbb{R}^{2}$ in such a way that $\operatorname{det} D \tilde{U} \geqslant 0$ on $\Omega$. Then by Müller's result, $\operatorname{det} D U \in L \log L(\mathbb{D})$. However (3.1) then implies $\nabla u_{1} \in L^{2} \log L(\mathbb{D})$ and hence $\varphi \in K_{1}(\mathbb{T})$ thus contradicting the choice of $\varphi$. We conclude that extensions such as $\tilde{U}$ are in general not possible. 
A simple application. The motivation of the following problem is from [8].

Consider the following boundary value problem:

$$
\begin{cases}\operatorname{det} D u(x)=f(x), & x \in \Omega, \\ u(x)=x, & x \in \partial \Omega,\end{cases}
$$

where $\Omega$ is a smooth domain in $\mathbb{R}^{n}$ and $f \geqslant 0$ is a measurable function satisfying $\int_{\Omega} f(x) d x=0$. We seek a necessary condition on $f$ to ensure that the problem is solvable for $u \in W^{1, n}\left(\Omega, \mathbb{R}^{n}\right)$.

COROLlaRY 3.4. - Suppose $u \in W^{1, n}\left(\Omega, \mathbb{R}^{n}\right)$ is a solution of problem $(*)$. Then $f \in L(\log L)(\Omega)$.

This is a direct consequence of Theorem 3.1. It leads to the following question.

Question. - Given $f \in L \log L(\Omega)$, does (*) have a solution $u \in$ $W^{1, n}(\Omega) ?$

Remark. - Theorems 2.5 and 3.1 can be easily generalized to the cases when $u=\left(u_{1}, u_{2}, \ldots, u_{n}\right) \in W^{1, n}\left(\Omega, \mathbb{R}^{n}\right)$ is replaced by $u_{j} \in$ $W^{1, p_{j}}\left(\Omega, \mathbb{R}^{n}\right), p_{j}>1, j=1,2, \ldots, n$, with $\sum_{j=1}^{n} \frac{1}{p_{j}}=1$.

\section{DIV-CURL RESULTS FOR FORMS}

The setting of this section is that of forms on open domains $\Omega \subset \mathbb{R}^{n}$. We give a brief outline of the basic formalism.

The space of $l$-linear, alternating functions $\xi:\left(\mathbb{R}^{n}\right)^{l} \rightarrow \mathbb{R}$ is denoted by $\Lambda^{l} \mathbb{R}^{n}$, or just $\Lambda^{l}$ when there is no possibility of confusion. In particular $\Lambda^{1} \mathbb{R}^{n}=\left(\mathbb{R}^{n}\right)^{\prime}$, the dual to $\mathbb{R}^{n}$ and $\Lambda^{0} \mathbb{R}^{n}=\mathbb{R}$. The exterior algebra of forms is denoted

$$
\Lambda\left(\mathbb{R}^{n}\right)=\bigoplus_{l=0}^{n} \Lambda^{l}\left(\mathbb{R}^{n}\right)
$$

and the wedge product of $\xi \in \Lambda^{l}$ and $\eta \in \Lambda^{k}$ is the $(k+l)$-form $\xi \wedge \eta$ given by

$$
(\xi \wedge \eta)\left(X_{1}, \ldots, X_{k+l}\right)=\sum \varepsilon(\sigma) \xi\left(X_{i_{1}}, \ldots, X_{i_{l}}\right) \eta\left(X_{j_{1}}, \ldots, X_{j_{k}}\right),
$$

where the sum is taken over all permutations $\sigma=\left\{i_{1}, \ldots, i_{l}, j_{1}, \ldots, j_{k}\right\}$ of $\{1, \ldots, k+l\}$ satisfying $i_{1}<\cdots<i_{l}$ and $j_{1}<\cdots<j_{k}$ and $\varepsilon(\sigma)$ is the 
signature of the permutation $\sigma$. The exterior product is alternating, i.e., $\xi \wedge \eta=-\eta \wedge \xi$. Fix a basis $\left\{e_{1}, \ldots, e_{n}\right\}$ for $\mathbb{R}^{n}$. An $r$-form is defined to be a function $u: \mathbb{R}^{n} \rightarrow \Lambda^{r}\left(\mathbb{R}^{n}\right)$ of the form

$$
u=\sum_{i_{1}, \ldots, i_{r}} u_{i_{1}, \ldots, i_{r}} e_{i_{1}} \wedge \cdots \wedge e_{i_{r}}
$$

where the sum is taken over subsets $\left\{i_{1}, \ldots, i_{r}\right\}$ of $\{1, \ldots, n\}$ and $u_{i_{1}, \ldots, i_{r}}$ are (real-valued) functions on $\mathbb{R}^{n}$. We denote by $L^{p}\left(\Omega, \Lambda^{r}\right)$ the space of $p$-integrable $r$-forms on $\Omega$.

The Hodge-deRham operator $d$ acts on smooth forms defined on $\Omega$ by

$$
d u=\sum_{k=1}^{n} \frac{\partial}{\partial x_{k}} e_{k} \wedge u
$$

and satisfies $d(d u)=0$.

In [6], it is proved that if $u \in L^{p}\left(\mathbb{R}^{n}, \mathbb{R}^{n}\right), \operatorname{div} u=0, v \in L^{q}\left(\mathbb{R}^{n}, \mathbb{R}^{n}\right)$, curl $v=0$, where $1 / p+1 / q=1$, then $u \cdot v \in H^{1}\left(\mathbb{R}^{n}\right)$. This is equivalent to the statement that, if $u \in L^{p}\left(\mathbb{R}^{n}, \Lambda^{n-1}\right), d u=0, v \in L^{q}\left(\mathbb{R}^{n}, \Lambda^{1}\right)$, $d v=0$, then $u \wedge v \in H^{1}\left(\mathbb{R}^{n}, \Lambda^{n}\right)$. More generally, the following is true.

Proposition 4.1. - If $1<p<\infty, 1 / p+1 / q=1, u \in L^{p}\left(\mathbb{R}^{n}, \Lambda^{k}\right)$, $v \in L^{q}\left(\mathbb{R}^{n}, \Lambda^{n-k}\right), d u=0, d v=0$ on $\mathbb{R}^{n}$, then $u \wedge v \in H^{1}\left(\mathbb{R}^{n}, \Lambda^{n}\right)$ and

$$
\|u \wedge v\|_{H^{1}} \leqslant c\|u\|_{L^{p}}\|v\|_{L^{q}} .
$$

Suppose $\Omega$ is a bounded open domain in $\mathbb{R}^{n}$ with strongly Lipschitz boundary. In this section, we provide the extra conditions on $u$ and $v$ which, together with $u \in L^{p}\left(\Omega, \Lambda^{n-1}\right), d u=0, v \in L^{q}\left(\Omega, \Lambda^{1}\right)$ and $d v=0$, imply that $u \wedge v \in h_{z}^{1}\left(\Omega, \Lambda^{n}\right)$.

To state and prove the theorem, we first introduce some notation and state several known results.

Stokes' theorem in this context is as follows: if $u \in C^{1}\left(\bar{\Omega}, \Lambda^{k}\right)$ and $\varphi \in C^{1}\left(\bar{\Omega}, \Lambda^{n-k-1}\right)$ then

$$
\begin{aligned}
\int_{\Omega} d u \wedge \varphi+(-1)^{k} \int_{\Omega} u \wedge d \varphi & =\int_{\partial \Omega} u \wedge \varphi=\int_{\partial \Omega} n \vee(n \wedge u \wedge \varphi) \\
& =\left\langle\left. n \wedge u\right|_{\partial \Omega}, \varphi\right\rangle_{\partial \Omega} .
\end{aligned}
$$

Here $n \vee(n \wedge u \wedge \varphi)$ is the tangential component of the $(n-1)$ form $u \wedge \varphi$ on $\partial \Omega$, while the final expression is the natural pairing between 
$(k+1)$ forms and $(n-k-1)$ forms on the boundary. This provides a natural meaning for the statement $d u=0$ :

DEFINITION 4.2. - Let $\mathcal{D}\left(\Omega, \Lambda^{n-k-1}\right)$ be the space of $n-k-1$ forms on $\mathbb{R}^{n}$ whose support is contained in $\Omega$. If $u \in L^{p}\left(\Omega, \Lambda^{k}\right)$, we say that $d u=0$ on $\Omega$ if $\int_{\Omega} u \wedge d \eta=0$ for all $\eta \in \mathcal{D}\left(\Omega, \Lambda^{n-k-1}\right)$.

DEFinition 4.3. - For those $u \in L^{p}\left(\Omega, \Lambda^{k}\right)$ with $d u=0$ on $\Omega$, define $\left.n \wedge u\right|_{\partial \Omega} \in W^{-1 / p, p}\left(\partial \Omega, \Lambda^{k+1}\right)$ by

$$
\left\langle\left. n \wedge u\right|_{\partial \Omega}, \varphi\right\rangle_{\partial \Omega}=(-1)^{k} \int_{\Omega} u \wedge d \Phi,
$$

where $\Phi \in C^{1}\left(\bar{\Omega}, \Lambda^{n-k-1}\right)$ and $\varphi=\left.\Phi\right|_{\partial \Omega}$.

It is a simple matter to show that the definition of $\left\langle\left. n \wedge u\right|_{\partial \Omega}, \varphi\right\rangle_{\partial \Omega}$ is independent of the choice of the extension $\Phi$. Note that

$$
\left\|\left.n \wedge u\right|_{\partial \Omega}\right\|_{W^{-1 / p, p}\left(\partial \Omega, \Lambda^{k+1}\right)} \leqslant c\|u\|_{L^{p}\left(\Omega, \Lambda^{k}\right)}
$$

for all $u \in L^{p}\left(\Omega, \Lambda^{k}\right)$ such that $d u=0$.

LEMMA 4.4. - Suppose $G \in W_{0}^{1, p}\left(\Omega, \Lambda^{k}\right)$. Then $\left.n \wedge d G\right|_{\partial \Omega}=0$.

Proof. - By the density of $C_{c}^{1}\left(\Omega, \Lambda^{k}\right)$ in $W_{0}^{1, p}\left(\Omega, \Lambda^{k}\right)$ and the preceding estimate, it is enough to consider $G \in C_{c}^{1}\left(\Omega, \Lambda^{k}\right)$. Then if $\Phi \in$ $C^{1}\left(\bar{\Omega}, \Lambda^{n-k-1}\right)$, and if $\varphi=\left.\Phi\right|_{\partial \Omega}$,

$$
\begin{aligned}
\left\langle\left. n \wedge d G\right|_{\partial \Omega}, \varphi\right\rangle_{\partial \Omega} & =(-1)^{k} \int_{\Omega} d G \wedge d \Phi \\
& =-\int_{\Omega} G \wedge d^{2} \Phi+\left\langle G,\left.n \wedge d \Phi\right|_{\partial \Omega}\right\rangle_{\partial \Omega}=0
\end{aligned}
$$

since $d^{2} \Phi=0$ and $\left.G\right|_{\partial \Omega}=0$.

We need several extension results. The first of these is as follows:

LEMMA 4.5. - Suppose $u \in L^{p}\left(\Omega, \Lambda^{k}\right), d u=0$ on $\Omega$ and $\left.n \wedge u\right|_{\partial \Omega}=$ 0 . Let $u_{z}$ be the zero extension of $u$ to $\mathbb{R}^{n}$. Then $d u_{z}=0$ on $\mathbb{R}^{n}$.

Proof. - If $\varphi \in C_{c}^{\infty}\left(\mathbb{R}^{n}, \Lambda^{n-k-1}\right)$, by Stokes' theorem we have

$$
\begin{aligned}
\int_{\mathbb{R}^{n}} d u_{z} \wedge \varphi & =(-1)^{k+1} \int_{\mathbb{R}^{n}} u_{z} \wedge d \varphi=(-1)^{k+1} \int_{\Omega} u \wedge d \varphi \\
& =-\left\langle\left. n \wedge u\right|_{\partial \Omega}, \varphi\right\rangle_{\partial \Omega}=0 .
\end{aligned}
$$


Hence $d u_{z}=0$ on $\mathbb{R}^{n}$.

DeFINITION 4.6. - Let $1 \leqslant p \leqslant \infty$ and $\alpha \geqslant 0$. Define

$$
W_{\alpha}^{1, p}(\Omega)=\left\{f \in W^{1, p}(\Omega) ; \frac{\partial f}{\partial x_{j}} \in L^{p}(\log L)^{\alpha}(\Omega) \text { for } j=1, \ldots, n\right\}
$$

and give it the seminorm

$$
\|f\|_{\dot{W}_{\alpha}^{1, p}(\Omega)}=\max _{j}\left\|\frac{\partial f}{\partial x_{j}}\right\|_{L^{p}(\log L)^{\alpha}(\Omega)} .
$$

When $\alpha=0$, this is written $\|f\|_{\dot{W}^{1, p}(\Omega)}$.

Definition 4.7. - Let $p, \alpha$ be as above. Then $W_{\alpha}^{1, p}\left(\Omega, \Lambda^{k}\right)$ is the class of $k$-forms $f$ each of whose components lies in $W_{\alpha}^{1, p}(\Omega)$.

We are now in a position to prove analogues of Proposition 4.1 on bounded domains. Let us first consider the case where certain boundary conditions are zero.

Proposition 4.8. - Suppose $1 \leqslant p<\infty, 1 / p+1 / q=1, u=d F \in$ $L^{p}\left(\Omega, \Lambda^{k}\right)$ for some $F \in W^{1, p}\left(\Omega, \Lambda^{k-1}\right), v \in L^{q}\left(\Omega, \Lambda^{n-k}\right)$ with $d v=0$ in $\Omega$ and $\left.n \wedge v\right|_{\partial \Omega}=0$. Then $u \wedge v \in H_{z}^{1}\left(\Omega, \Lambda^{n}\right)$ and

$$
\|u \wedge v\|_{H_{z}^{1}} \leqslant c\|v\|_{L^{q}}\|F\|_{\dot{W}^{1, p}} .
$$

Proof. - Without loss of generality, we may assume that each component $F_{j}$ of $F$ satisfies $\int_{\Omega} F_{j}=0$. Since $F \in W^{1, p}\left(\Omega, \Lambda^{k-1}\right)$, it may be extended to $\mathbb{R}^{n}$ with

$$
\|F\|_{W^{1, p}\left(\mathbb{R}^{n}, \Lambda^{k-1}\right)} \leqslant c\|F\|_{W^{1, p}\left(\Omega, \Lambda^{k-1}\right)} .
$$

Let $u=d F$ on $\mathbb{R}^{n}$. Then $d u=0$. By Lemma 4.5 , the zero extension $v_{z}$ of $v$ to $\mathbb{R}^{n}$ satisfies $d v_{z}=0$. By Proposition 4.1, the extensions of $u$ and $v$ satisfy $u \wedge v_{z} \in H^{1}\left(\mathbb{R}^{n}, \Lambda^{n}\right)$ and since $u \wedge v_{z}=0$ outside $\Omega$ we have

$$
\begin{aligned}
\|u \wedge v\|_{H_{z}^{1}\left(\Omega, \Lambda^{n}\right)} & =\left\|u \wedge v_{z}\right\|_{H_{z}^{1}\left(\mathbb{R}^{n}, \Lambda^{n}\right)} \leqslant c\|u\|_{L^{p}\left(\mathbb{R}^{n}\right)}\left\|v_{z}\right\|_{L^{q}\left(\mathbb{R}^{n}\right)} \\
& =c\|d F\|_{L^{p}\left(\mathbb{R}^{n}\right)}\|v\|_{L^{q}(\Omega)} \leqslant c\|F\|_{W^{1, p}\left(\mathbb{R}^{n}, \Lambda^{k-1}\right)}\|v\|_{L^{q}(\Omega)} \\
& \leqslant c\|F\|_{\dot{W}^{1, p}\left(\mathbb{R}^{n}, \Lambda^{k-1}\right)}\|v\|_{L^{q}(\Omega)},
\end{aligned}
$$

where in the last step we have used the Poincaré inequality.

In the special case $k=1$, this can be written in more classical notation as follows. 
COROLlary 4.9. - Suppose $1 \leqslant p<\infty, 1 / p+1 / q=1, \quad F \in$ $W^{1, p}(\Omega), u=\nabla F, v \in L^{q}\left(\Omega, \mathbb{R}^{n}\right), \operatorname{div} v=0$ on $\Omega$ and $\left.n \cdot v\right|_{\partial \Omega}=0$. Then $u \cdot v \in H_{z}^{1}(\Omega)$ and

$$
\|u \cdot v\|_{H_{z}^{1}} \leqslant c\|u\|_{L^{p}}\|v\|_{L^{q}} .
$$

We turn now to general boundary conditions.

DeFINITION 4.10. - Let $p, \alpha$ be as above. Then $K_{\alpha}^{p}\left(\partial \Omega, \Lambda^{k}\right)$ is the class of those $f \in W^{1-1 / p, p}\left(\partial \Omega, \Lambda^{k}\right)$ for which there exists $F \in$ $W_{\alpha}^{1, p}\left(\Omega, \Lambda^{k}\right)$ with $\left.F\right|_{\partial \Omega}=f$. It is given the seminorm

$$
\|f\|_{K_{\alpha}^{p}\left(\partial \Omega, \Lambda^{k}\right)}=\inf \left\{\|F\|_{\dot{W}_{\alpha}^{1, p}\left(\Omega, \Lambda^{k}\right)} ;\left.\quad F\right|_{\partial \Omega}=f\right\} .
$$

Suppose $f \in W^{1-1 / p, p}\left(\partial \Omega, \Lambda^{k}\right)$. Define $(n \wedge d) f \in W^{-1 / p, p}(\partial \Omega$, $\Lambda^{k+2}$ ) by

$$
(n \wedge d) f=\left.n \wedge d F\right|_{\partial \Omega},
$$

where $F \in W^{1, p}\left(\Omega, \Lambda^{k}\right)$ is an extension of $f$ to $\Omega$, i.e., $\left.F\right|_{\partial \Omega}=f$. That this definition is independent of the choice of extension $F$ is a consequence of Lemma 4.4.

Definition 4.11. - Let $p, \alpha$ be as above. Define $J_{\alpha}^{p}\left(\partial \Omega, \Lambda^{k}\right)=$ $(n \wedge d) K_{\alpha}^{p}\left(\partial \Omega, \Lambda^{k-2}\right)$ with

$$
\|g\|_{J_{\alpha}^{p}\left(\partial \Omega, \Lambda^{k}\right)}=\inf \left\{\|f\|_{K_{\alpha}^{p}\left(\partial \Omega, \Lambda^{k-2}\right)} ; g=(n \wedge d) f\right\} .
$$

A second extension result, this time for extensions from $\partial \Omega$ to $\Omega$, follows.

PROPOSITION 4.12. - Let $p, \alpha$ be as above and let $g \in J_{\alpha}^{p}\left(\partial \Omega, \Lambda^{k}\right)$. Then there exists $F \in W^{1, p}\left(\mathbb{R}^{n}, \Lambda^{k-1}\right)$ such that $\left.n \wedge d F\right|_{\partial \Omega}=g,\left.F\right|_{\Omega} \in$ $W_{\alpha}^{1, p}\left(\Omega, \Lambda^{k-1}\right)$ and

$$
\|F\|_{\dot{W}^{1, p}\left(\mathbb{R}^{n}, \Lambda^{k-1}\right)} \leqslant c\|F\|_{\dot{W}_{\alpha}^{1, p}\left(\Omega, \Lambda^{k-1}\right)} \leqslant c\|g\|_{J_{\alpha}^{p}\left(\partial \Omega, \Lambda^{k}\right)} .
$$

Proof. - The proof is simply a matter of checking definitions. Since $g \in J_{\alpha}^{p}\left(\partial \Omega, \Lambda^{k}\right)$, there exists $f \in K_{\alpha}^{p}\left(\partial \Omega, \Lambda^{k-2}\right)$ with $g=(n \wedge d) f$ and

$$
\|f\|_{K_{\alpha}^{p}\left(\partial \Omega, \Lambda^{k-2}\right)} \leqslant 2\|g\|_{J_{\alpha}^{p}\left(\partial \Omega, \Lambda^{k}\right)} .
$$


Since $f \in K_{\alpha}^{p}\left(\partial \Omega, \Lambda^{k-2}\right)$, there exists $F \in W_{\alpha}^{1, p}\left(\Omega, \Lambda^{k-2}\right)$ with $\left.F\right|_{\Omega}=$ $f$ and

$$
\|F\|_{\dot{W}_{\alpha}^{1, p}\left(\Omega, \Lambda^{k-2}\right)} \leqslant 2\|f\|_{K_{\alpha}^{p}\left(\partial \Omega, \Lambda^{k-2}\right)} .
$$

Without loss of generality, by adding a constant $(k-2)$-form if necessary, we may assume that $\int_{\Omega} F=0$. Also, $g=(n \wedge d) f=\left.n \wedge d F\right|_{\partial \Omega}$ by the definition of $(n \wedge d) f$ and its independence from the choice of the extension of $f$. Further, $F \in W^{1, p}\left(\Omega, \Lambda^{k-2}\right)$ and since $\partial \Omega$ is Lipschitz, $F$ can be extended to $\mathbb{R}^{n}$ with

$$
\begin{aligned}
\|F\|_{\dot{W}^{1, p}\left(\mathbb{R}^{n}, \Lambda^{k-2}\right)} & \leqslant\|F\|_{W^{1, p}\left(\mathbb{R}^{n}, \Lambda^{k-2}\right)} \leqslant c\|F\|_{W^{1, p}\left(\Omega, \Lambda^{k-2}\right)} \\
& \leqslant c\|F\|_{\dot{W}_{\alpha}^{1, p}\left(\Omega, \Lambda^{k-2}\right)},
\end{aligned}
$$

where the last step is a consequence of the Poincaré inequality.

The main result of this section is:

TheOREM 4.13. - Suppose $1<p<\infty, \alpha, \beta \geqslant 0, \alpha / p+\beta / q=1$, $u \in L^{p}\left(\Omega, \Lambda^{k}\right), v \in L^{q}\left(\Omega, \Lambda^{n-k}\right), d u=0, d v=0$ on $\Omega$. Suppose also that $\left.n \wedge u\right|_{\partial \Omega} \in J_{\alpha}^{p}\left(\partial \Omega, \Lambda^{k+1}\right)$ and $\left.n \wedge v\right|_{\partial \Omega} \in J_{\beta}^{q}\left(\partial \Omega, \Lambda^{n-k+1}\right)$. Then $u \wedge v \in h_{z}^{1}\left(\Omega, \Lambda^{n}\right)$ and

$$
\|u \wedge v\|_{h_{z}^{1}} \leqslant c\left(\|u\|_{L^{p}}+\left\|\left.n \wedge u\right|_{\partial \Omega}\right\|_{J_{\alpha}^{p}}\right)\left(\|v\|_{L^{q}}+\left\|\left.n \wedge v\right|_{\partial \Omega}\right\|_{J_{\beta}^{q}}\right) .
$$

Proof. - By Proposition 4.12, since $\left.n \wedge u\right|_{\partial \Omega} \in J_{\alpha}^{p}\left(\partial \Omega, \Lambda^{k+1}\right)$ and $\left.n \wedge v\right|_{\partial \Omega} \in J_{\beta}^{q}\left(\partial \Omega, \Lambda^{n-k+1}\right)$, there exist $F \in W^{1, p}\left(\mathbb{R}^{n}, \Lambda^{k-1}\right)$ and $G \in$ $W^{1, q}\left(\mathbb{R}^{n}, \Lambda^{n-k-1}\right)$ such that $\left.n \wedge d F\right|_{\partial \Omega}=n \wedge u,\left.n \wedge d G\right|_{\partial \Omega}=n \wedge v$, $\left.F\right|_{\Omega} \in W_{\alpha}^{1, p}\left(\Omega, \Lambda^{k-1}\right),\left.G\right|_{\Omega} \in W_{\beta}^{1, p^{\prime}}\left(\Omega, \Lambda^{n-k-1}\right)$ and

$$
\begin{gathered}
\|F\|_{\dot{W}^{1, p}\left(\mathbb{R}^{n}, \Lambda^{k-1}\right)} \leqslant c\|F\|_{\dot{W}^{1, p}\left(\Omega, \Lambda^{k-1}\right)} \leqslant c\left\|\left.n \wedge u\right|_{\partial \Omega}\right\|_{J_{\alpha}^{p}\left(\partial \Omega, \Lambda^{k+1}\right)}, \\
\|G\|_{\dot{W}^{1, q}\left(\mathbb{R}^{n}, \Lambda^{n-k-1}\right)} \leqslant c\|G\|_{\dot{W}^{1, q}\left(\Omega, \Lambda^{n-k-1}\right)} \leqslant c\left\|\left.n \wedge v\right|_{\partial \Omega}\right\|_{J_{\beta}^{q}\left(\partial \Omega, \Lambda^{n-k+1}\right)} .
\end{gathered}
$$

Also $d(u-d F)=d u-d^{2} F=0$ on $\Omega$ and $\left.n \wedge(u-d F)\right|_{\partial \Omega}=\left.n \wedge u\right|_{\partial \Omega}-$ $\left.n \wedge d F\right|_{\partial \Omega}=0$. Similarly, $d(v-d G)=0$ on $\Omega$ and $\left.n \wedge(v-d G)\right|_{\partial \Omega}=0$. So $(u-d F)_{z}$, the zero extension of $u-d F$ to $\mathbb{R}^{n}$ satisfies $d\left((u-d F)_{z}\right)=$ 0 on $\mathbb{R}^{n}$ and

$$
\begin{aligned}
& \left\|(u-d F)_{z}\right\|_{L^{p}\left(\mathbb{R}^{n}, \Lambda^{k}\right)} \\
& \quad=\|u-d F\|_{L^{p}\left(\Omega, \Lambda^{k}\right)} \leqslant\|u\|_{L^{p}\left(\Omega, \Lambda^{k}\right)}+\|F\|_{\dot{W}^{1, p}\left(\Omega, \Lambda^{k}\right)} \\
& \quad \leqslant\|u\|_{L^{p}\left(\Omega, \Lambda^{k}\right)}+\|F\|_{\dot{W}_{\alpha}^{1, p}\left(\Omega, \Lambda^{k}\right)} \\
& \quad \leqslant c\left(\|u\|_{L^{p}\left(\Omega, \Lambda^{k}\right)}+\left\|\left.n \wedge u\right|_{\partial \Omega}\right\|_{J_{\alpha}^{p}\left(\partial \Omega, \Lambda^{k+1}\right)}\right) .
\end{aligned}
$$


Similarly, $(v-d G)_{z}$ satisfies $d\left((v-d G)_{z}\right)=0$ on $\mathbb{R}^{n}$ and

$$
\left\|(v-d G)_{z}\right\|_{L^{q}\left(\mathbb{R}^{n}, \Lambda^{n-k}\right)} \leqslant c\left(\|v\|_{L^{q}\left(\Omega, \Lambda^{n-k}\right)}+\left\|\left.n \wedge v\right|_{\partial \Omega}\right\|_{J_{\beta}^{q}\left(\partial \Omega, \Lambda^{n-k+1)}\right)}\right) .
$$

Let $U=d F$ on $\mathbb{R}^{n}$. Then $U \in L^{p}\left(\mathbb{R}^{n}, \Lambda^{k}\right)$, and

$$
\begin{aligned}
\|U\|_{L^{p}\left(\mathbb{R}^{n}, \Lambda^{k}\right)} & =\|d F\|_{L^{p}\left(\mathbb{R}^{n}, \Lambda^{k}\right)} \leqslant c\|F\|_{\dot{W}^{1, p}\left(\mathbb{R}^{n}, \Lambda^{k-1}\right)} \\
& \leqslant c\left\|\left.n \wedge u\right|_{\partial \Omega}\right\|_{J_{\alpha}^{p}\left(\partial \Omega, \Lambda^{k+1}\right)} .
\end{aligned}
$$

Let $V=d G$ on $\mathbb{R}^{n}$. Then $V \in L^{q}\left(\mathbb{R}^{n}, \Lambda^{n-k}\right)$, and

$$
\|V\|_{L^{q}\left(\mathbb{R}^{n}, \Lambda^{n-k}\right)} \leqslant c\left\|\left.n \wedge v\right|_{\partial \Omega}\right\|_{J_{\beta}^{q}\left(\partial \Omega, \Lambda^{n-k+1}\right)} .
$$

Now let $\tilde{u}=u \chi_{\Omega}+U \chi_{\Omega^{\prime}}$ where $\chi_{\Omega}$ is the characteristic function of $\Omega$ and $\chi_{\Omega^{\prime}}=1-\chi_{\Omega}$. Then

$$
\begin{aligned}
\|\tilde{u}\|_{L^{p}\left(\mathbb{R}^{n}, \Lambda^{k}\right)} & =\|U\|_{L^{p}\left(\Omega^{\prime}, \Lambda^{k}\right)}+\|u\|_{L^{p}\left(\Omega, \Lambda^{k}\right)} \\
& \leqslant c\left(\|u\|_{L^{p}\left(\Omega, \Lambda^{k}\right)}+\left\|\left.n \wedge u\right|_{\partial \Omega}\right\|_{J_{\alpha}^{p}\left(\partial \Omega, \Lambda^{k+1}\right)}\right) .
\end{aligned}
$$

Also, we may write $\tilde{u}=U+(u-U)_{z}$, from which we see easily that $d \tilde{u}=0$ on $\mathbb{R}^{n}$. Similarly, let $\tilde{v}=v \chi_{\Omega}+V \chi_{\Omega^{\prime}}$. Then

$$
\|\tilde{v}\|_{L^{q}\left(\mathbb{R}^{n}, \Lambda^{n-k}\right)} \leqslant c\left(\|v\|_{L^{q}\left(\Omega, \Lambda^{n-k}\right)}+\left\|\left.n \wedge v\right|_{\partial \Omega}\right\|_{J_{\beta}^{q}\left(\partial \Omega, \Lambda^{n-k+1}\right)}\right)
$$

and $d \tilde{v}=0$ on $\mathbb{R}^{n}$. Now we write, on $\Omega$,

$$
\begin{aligned}
u \wedge v & =u \wedge(v-V)+(u-U) \wedge V+U \wedge V \\
& =\tilde{u} \wedge(v-V)_{z}+(u-U)_{z} \wedge V+U \wedge V .
\end{aligned}
$$

To deal with the first term on the right hand side of (4.1), notice that $\tilde{u} \in L^{p}\left(\mathbb{R}^{n}, \Lambda^{k}\right),(v-V)_{z} \in L^{q}\left(\mathbb{R}^{n}, \Lambda^{n-k}\right), d \tilde{u}=0$ on $\mathbb{R}^{n}$ and $d((v-$ $\left.V)_{z}\right)=0$ on $\mathbb{R}^{n}$. Hence, by an application of Proposition 4.1, we have $\tilde{u} \wedge(v-V)_{z} \in H^{1}\left(\mathbb{R}^{n}, \Lambda^{n}\right)$ with the bound

$$
\begin{aligned}
& \left\|\tilde{u} \wedge(v-V)_{z}\right\|_{H^{1}} \leqslant c\|\tilde{u}\|_{L^{p}}\left\|(v-V)_{z}\right\|_{L^{q}} \\
& \quad \leqslant c\left(\|u\|_{L^{p}}+\left\|\left.n \wedge u\right|_{\partial \Omega}\right\|_{J_{\alpha}^{p}}\right)\left(\|v\|_{L^{q}}+\left\|\left.n \wedge v\right|_{\partial \Omega}\right\|_{J_{\beta}^{q}}\right) .
\end{aligned}
$$

Similarly for the second term on the right hand side of (4.1) we have $(u-U)_{z} \in L^{p}\left(\mathbb{R}^{n}, \Lambda^{k}\right), V \in L^{q}\left(\mathbb{R}^{n}, \Lambda^{n-k}\right), d\left((u-U)_{z}\right)=0$ on $\mathbb{R}^{n}$ and $d V=0$ on $\mathbb{R}^{n}$, so again by Proposition 4.1 we have $(u-U)_{z} \wedge V \in$ $H^{1}\left(\mathbb{R}^{n}, \Lambda^{n}\right)$ and 


$$
\begin{aligned}
\left\|(u-U)_{z} \wedge V\right\|_{H^{1}} & \leqslant c\left\|(u-U)_{z}\right\|_{L^{p}}\|V\|_{L^{q}} \\
& \leqslant c\left(\|u\|_{L^{p}}+\left\|\left.n \wedge u\right|_{\partial \Omega}\right\|_{J_{\alpha}^{p}}\right)\left\|\left.n \wedge v\right|_{\partial \Omega}\right\|_{J_{\beta}^{q}} .
\end{aligned}
$$

Finally, since $U=d F \in L^{p}(\log L)^{\alpha}\left(\Omega, \Lambda^{k}\right), V=d G \in L^{q}(\log L)^{\beta}$ $\left(\Omega, \Lambda^{n-k}\right)$ and $\int_{\Omega} U \wedge V=0$, we have $U \wedge V \in L \log L\left(\Omega, \Lambda^{n}\right) \subset$ $h_{z}^{1}\left(\Omega, \Lambda^{n}\right)$ and

$$
\begin{aligned}
\|U \wedge V\|_{h_{z}^{1}} & \leqslant c\|U \wedge V\|_{L \log L(\Omega)} \\
& \leqslant c\|U\|_{L^{p}(\log L)^{\alpha}\left(\Omega, \Lambda^{k}\right)}\|V\|_{L^{q}(\log L)^{\beta}\left(\Omega, \Lambda^{n-k}\right)} \\
& \leqslant c\|F\|_{\dot{W}_{\alpha}^{1, p}\left(\Omega, \Lambda^{k}\right)}\|G\|_{\dot{W}_{\beta}^{1, q}\left(\Omega, \Lambda^{n-k}\right)} \\
& \leqslant c\|n \wedge u\|_{J_{\alpha}^{p}\left(\partial \Omega, \Lambda^{k+1}\right)}\|n \wedge v\|_{J_{\beta}^{q}\left(\partial \Omega, \Lambda^{n-k+1}\right)}
\end{aligned}
$$

Combining Eqs. (4.1)-(4.4) now gives the result.

Note that Proposition 4.8 can be obtained from this theorem on choosing $\alpha=0, \beta=q$.

The discussion of weak continuity and higher integrability of this bilinear differential form is very similar to that of Jacobians. We will skip the details and only state the results.

THEOREM 4.14. - Let $1<p<\infty, \alpha, \beta \geqslant 0$, and $\alpha / p+\beta / q=1$. Suppose $u_{j} \in L^{p}\left(\Omega, \Lambda^{k}\right)$ is a bounded sequence, $d u_{j}=0$ and $u_{j} \rightarrow u$ weakly in $L^{p}\left(\Omega, \Lambda^{k}\right), v_{j} \in L^{q}\left(\Omega, \Lambda^{n-k}\right)$ is a bounded sequence, $d v_{j}=0$ and $v_{j} \rightarrow v$ weakly in $L^{q}\left(\Omega, \Lambda^{k}\right)$. If $\left.n \wedge u_{j}\right|_{\partial \Omega} \in J_{\alpha}^{p}\left(\partial \Omega, \Lambda^{k+1}\right)$ and $\left.n \wedge v_{j}\right|_{\partial \Omega} \in J_{\beta}^{q}\left(\partial \Omega, \Lambda^{n-k+1}\right)$ are bounded sequences, then up to a subsequence,

$$
\int_{\Omega} b u_{j} \wedge v_{j} \rightarrow \int_{\Omega} b u \wedge v
$$

for all $b \in \operatorname{VMO}\left(\Omega, \Lambda^{0}\right)$.

THEOREM 4.15. - Assume $1<p<\infty, \alpha, \beta \geqslant 0, \alpha / p+\beta / q=1$, $u \in L^{p}\left(\Omega, \Lambda^{k}\right), v \in L^{q}\left(\Omega, \Lambda^{n-k}\right), d u=0, d v=0$ on $\Omega$ and $u \wedge$ $v \geqslant 0$. Suppose also that $\left.n \wedge u\right|_{\partial \Omega} \in J_{\alpha}^{p}\left(\partial \Omega, \Lambda^{k+1}\right)$ and $\left.n \wedge v\right|_{\partial \Omega} \in$ $J_{\beta}^{q}\left(\partial \Omega, \Lambda^{n-k+1}\right)$. Then $u \wedge v \in L \log L\left(\Omega, \Lambda^{n}\right)$ and

$$
\|u \wedge v\|_{L \log L} \leqslant c\left(\|u\|_{L^{p}}+\left\|\left.n \wedge u\right|_{\partial \Omega}\right\|_{J_{\alpha}^{p}}\right)\left(\|v\|_{L^{q}}+\left\|\left.n \wedge v\right|_{\partial \Omega}\right\|_{J_{\beta}^{q}}\right) .
$$




\section{A. APPENDIX}

This section is devoted to the proof of a generalised Hölder inequality, of which Proposition 2.2 is a particular case, and to the proof of Proposition 3.2. The proofs of Theorem A.1 and Lemma A.2 are an amalgam of arguments found in [18], [15] and [16] and from private correspondence between Stephen Montgomery-Smith, Richard O'Neil and the authors.

Theorem A.1. - Suppose A, $B, C:[0, \infty) \rightarrow[0, \infty)$ are continuous, monotone increasing functions for which there exist positive constants $c$ and $d$ such that

(i) $B^{-1}(t) C^{-1}(t) \leqslant c A^{-1}(t)$ for all $t>0$, and

(ii) $A\left(\frac{t}{d}\right) \leqslant \frac{1}{2} A(t)$ for all $t>0$.

Suppose also that $\Omega$ is an open subset of $\mathbb{R}^{n}, f \in L_{B}(\Omega)$ and $g \in$ $L_{C}(\Omega)$. Then $f g \in L_{A}(\Omega)$ and

$$
\|f g\|_{A} \leqslant c d\|f\|_{B}\|g\|_{C} .
$$

As a preliminary to the proof of the theorem, we have the following lemma:

Lemma A.2. - Let $A, B$ and $C$ be as above. Then, for all $s, t>0$,

$$
A\left(\frac{s t}{c}\right) \leqslant B(s)+C(t)
$$

Proof. - Let $u=B(s)$ and $v=C(t)$. Then

$$
s t=B^{-1}(u) C^{-1}(v) \leqslant B^{-1}(u+v) C^{-1}(u+v) \leqslant c A^{-1}(u+v) .
$$

Dividing by $c$ and applying $A$ to both sides gives the result.

Proof of Theorem A.1. - Note that if $f \in L_{A}(\Omega)$, the monotonicity of $A$ and an application of the monotone convergence theorem gives us that $\int_{\Omega} A\left(|f(x)| /\|f\|_{A}\right) d x \leqslant 1$. Hence, from the definition of the Luxemburg norm

$$
\int_{\Omega} A\left(\frac{f(x) g(x)}{c\|f\|_{B}\|g\|_{C}}\right) d x \leqslant \int_{\Omega} B\left(\frac{f(x)}{\|f\|_{B}}\right) d x+\int_{\Omega} C\left(\frac{g(x)}{\|g\|_{C}}\right) d x \leqslant 2 .
$$


We therefore have

$$
\int_{\Omega} A\left(\frac{f(x) g(x)}{c d\|f\|_{B}\|g\|_{C}}\right) d x \leqslant 1
$$

and, again by the definition of the Luxemburg norm, we have the result.

Proof of Proposition 2.2. - To prove the generalised Hölder inequality of Proposition 2.2, we need only show that if $B(s)=s^{p} \log ^{\alpha}(e+s)$, then $B^{-1}(t) \approx t^{1 / p}(\log (e+t))^{-\alpha / p}$. To see this, simply note that if $t=s^{p}(\log (e+s))^{\alpha}$, there exist constants $0<c_{1}(p, \alpha) \leqslant c_{2}(p, \alpha)<\infty$ such that for all $s>0$,

$$
c_{1}(p, \alpha) \log (e+t) \leqslant \log (e+s) \leqslant c_{2}(p, \alpha) \log (e+t) .
$$

Then $t=s^{p}(\log (e+s))^{\alpha} \approx s^{p}(\log (e+t))^{\alpha}$ and solving for $s$ gives $s \approx t^{1 / p}(\log (e+t))^{-\alpha / p}$. This completes the proof.

The proof of Proposition 3.2 relies on well-known facts about maximal functions and Hardy space which we now collect.

LEMMA A.3. - Let $f$ be supported in a ball $B \subset \mathbb{R}^{n}$ and let $M f$ be its Hardy-Littlewood maximal function. Then $f \in L \log L(B)$ if and only if $M f \in L^{1}(B)$. Furthermore, there exist constant $c_{1}$ and $c_{2}$ independent of $f$ for which

$$
c_{2}\|M f\|_{L^{1}(B)} \leqslant\|f\|_{L \log L(B)} \leqslant c_{1}\|M f\|_{L^{1}(B)} .
$$

For a proof of this result, the reader is referred to [22] or [23, Chapter 1, Section 5.2, p. 23].

The space $H^{1}\left(\mathbb{R}^{n}\right)$ is defined in terms of the so-called "grand maximal function":

$$
f^{*}(x)=\sup _{t>0} \sup _{\varphi \in \mathcal{T}} \frac{1}{t^{n}}\left|\int_{\mathbb{R}^{n}} \varphi\left(\frac{x-y}{t}\right) f(y) d y\right|,
$$

where $\mathcal{T}=\left\{\varphi \in C^{\infty}\left(\mathbb{R}^{n}\right) ; \operatorname{supp} \varphi \subset B(0,1),\|\nabla \varphi\|_{\infty} \leqslant 1\right\}$ and $B(0,1)=$ $\left\{y \in \mathbb{R}^{n}:|y| \leqslant 1\right\}$. For further information, the reader is referred to [21]. A distribution $f$ on $\mathbb{R}^{n}$ lies in $H^{1}\left(\mathbb{R}^{n}\right)$ if $f^{*} \in L^{1}\left(\mathbb{R}^{n}\right)$ and $\|f\|_{H^{1}\left(\mathbb{R}^{n}\right)}=$ $\left\|f^{*}\right\|_{L^{1}\left(\mathbb{R}^{n}\right)}$. While it is always true that $f^{*} \leqslant c M f$, we also have $M f \leqslant$ $c f^{*}$ when $f \geqslant 0$. 
Proof of Proposition 3.2. - Let $K=\frac{1}{|\Omega|}\left|\int_{\Omega} f\right|$ and $\chi_{B}$ be the characteristic function of a ball $B$ containing $\bar{\Omega}$. Notice that by the normalisation on the test functions in $\mathcal{T},\left\|\left(\chi_{B}\right)^{*}\right\|_{\infty}$ is bounded, and hence that $\left\|\left(\chi_{B}\right)^{*}\right\|_{L^{1}(B)} \leqslant c|B|$. If we put $F=f-K$ on $\Omega$ and extend $F$ by zero off $\Omega$, then $f=F+K$ and $F \in H_{z}^{1}(\Omega)$. Also, $F+K \chi_{B} \geqslant 0$ on $B$, so

$$
\begin{aligned}
\|f\|_{L \log L(\Omega)} & =\|F+K\|_{L \log L(\Omega)} \\
& \leqslant c\left\|F+K \chi_{B}\right\|_{L \log L(B)} \\
& \leqslant c\left\|M\left(F+K \chi_{B}\right)\right\|_{L^{1}(B)} \quad(\text { by Lemma A.3) } \\
& \leqslant c\left\|\left(F+K \chi_{B}\right)^{*}\right\|_{L^{1}(B)} \quad\left(\text { since }\left.\left(F+K \chi_{B}\right)\right|_{B} \geqslant 0\right) \\
& \leqslant c\left\|F^{*}\right\|_{L^{1}\left(\mathbb{R}^{n}\right)}+K\left\|\left(\chi_{B}\right)^{*}\right\|_{L^{1}(B)} \\
& \leqslant c\|F\|_{H^{1}(\Omega)}+c K|B| \\
& \leqslant c \Omega\|f\|_{h_{z}^{1}(\Omega)} .
\end{aligned}
$$

\section{REFERENCES}

[1] Adams R.A., Sobolev Spaces, Academic Press, New York, 1975.

[2] Ball J.M., Murat F., $W^{1, p}$-quasiconvexity and variational problems for multiple integrals, J. Funct. Anal. 58 (1984) 225-253.

[3] Bennett C., Sharpley R., Interpolation of Operators, Academic Press, Boston, 1988.

[4] Brézis H., Fusco N., Sbordone C., Integrability for the Jacobian of orientationpreserving mappings, J. Funct. Anal. 115 (2) (1993) 425-431.

[5] Chang D.-C., Krantz S.G., Stein E.M., $H^{p}$ Theory on a smooth domain in $\mathbb{R}^{N}$ and elliptic boundary problems, J. Funct. Anal. 114 (1993) 286-347.

[6] Coifman R., Lions P.-L., Meyer Y., Semmes S., Compensated compactness and Hardy spaces, J. Math. Pures Appl. 72 (1993) 247-286.

[7] Dacorogna B., Weak continuity and weak lower semicontinuity of nonlinear functionals, in: Lect. Notes Math., Vol. 922, Springer, Berlin, 1982.

[8] Dacorogna B., Moser J., On a partial differential equation involving the Jacobian determinant, Ann. Inst. H. Poincaré Anal. Non Lineaire 7 (1990) 1-26.

[9] Ekeland I., Temam R., Convex Analysis and Variational Problems, North-Holland, Amsterdam, 1976.

[10] Greco L., Iwaniec T., Moscariello G., Limits on the improved integrability of the volume forms, Indiana Univ. Math. J. 44 (1995) 305-339.

[11] Iwaniec T., Integrability theory of the Jacobians, Vorlesungsreihe Rheinische Friedrich-Wilhelms-Universität Bonn 36 (1995).

[12] Iwaniec T., Sbordone C., On the integrability of the Jacobian under minimal hypotheses, Arch. Rational Mech. Anal. 119 (1992) 129-143.

[13] Jones P., Journé J.L., On weak convergence in $\mathcal{H}^{1}\left(R^{d}\right)$, Proc. Amer. Math. Soc. 120 (1994) 137-138.

[14] Lacroix M.-T., Espaces de traces ses espaces de Sobolev-Orlicz, J. de Math. Pures et Appl. 53 (1974) 439-458. 
[15] Montgomery-Smith S., The cotype of operators from $C(K)$, Ph.D. Thesis, Cambridge, 1989.

[16] Montgomery-Smith S., Comparison of Orlicz-Lorentz spaces, Studia Math. 103 (1993) 161-189.

[17] Müller S., Higher integrability of determinants and weak convergence in $L^{1}$, J. Reine Angew. Math. 412 (1990) 20-34.

[18] O’Neil R., Fractional integration in Orlicz spaces. I, Trans. Amer. Math. Soc. 115 (1965) 300-328.

[19] Robbin J.W., Rogers R.C., Temple B., On weak continuity and the Hodge decomposition, Trans. Amer. Math. Soc. 303 (1987) 609-618.

[20] Rogers R.C., Temple B., A characterization of the weakly continuous polynomials in the method of compensated compactness, Trans. Amer. Math. Soc. 310 (1988) 405-417.

[21] Semmes S., A primer on Hardy spaces, and some remarks on a theorem of Evans and Müller, Comm. Partial Differential Equations 19 (1994) 277-319.

[22] Stein E.M., Note on the class $L \log L$, Studia Math. 32 (1969) 305-310.

[23] Stein E.M., Singular Integrals and Differentiability Properties of Functions, Princeton University Press, Princeton, 1970.

[24] Ye D., Prescribing the Jacobian determinant in Sobolev spaces, Ann. Inst. Henri Poincare (Analyse non lineaire) 11 (3) (1994) 275-296. 